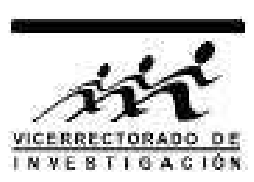

\title{
Fuente sísmica del terremoto de Huacho-Perú 1966 de 8.1 Mw a partir de inversión de registros mareográficos
}

\author{
C. Jiménez*1,2, N. Moggiano ${ }^{1,2}$, S. Yauri ${ }^{3}$ y M. Calvo ${ }^{1}$ \\ ${ }^{1}$ Facultad de Ciencias Físicas, Universidad Nacional Mayor de San Marcos, Lima, Perú \\ ${ }^{2}$ Dirección de Hidrografía y Navegación, Marina de Guerra del Perú, Callao, Perú \\ ${ }^{3}$ Instituto Nacional de Defensa Civil, INDECI, Lima, Perú
}

Recibido 22 marzo 2016 - Aceptado 6 mayo 2016

\begin{abstract}
El 17 de octubre de 1966 a las 21:41 UTC, un fuerte sismo remeció la región central del Perú, ocasionando 100 víctimas mortales. La máxima severidad de sacudimiento se reportó en la ciudad de Huacho (VIII a IX MM). Como efecto cosísmico se produjo un maremoto que inundó algunos poblados y balnearios costeros, como Casma y Tortugas. A partir del análisis y procesamiento de tres señales mareográficas registradas en las estaciones de Chimbote, Callao y Marcona, se determinaron los parámetros de la fuente sísmica mediante un proceso de inversión, en el que se comparó la señal simulada con la señal observada usando el método de mínimos cuadrados no-negativos. La máxima dislocación, slip, fue de $4.7 \mathrm{~m}$ ubicada en la parte sur del área de ruptura, lo que implica que la máxima aspereza o zona de mayor liberación de energía se encuentra ubicada en el océano frente a la ciudad de Huacho, lo que concuerda con los máximos valores de intensidad reportados. El momento tsunamigenico escalar obtenido fue de $M_{0}=1.76 \times 10^{21} \mathrm{Nm}$, lo que equivale a una magnitud de $8.1 \mathrm{Mw}$.
\end{abstract}

Palabras claves: Terremoto, maremoto, inversión, simulación numérica.

\section{Seismic source of the 1966 Huacho-Peru 8.1 Mw earthquake from tsunami waveform in- version}

On October 17, 1966 at 21:41 UTC, a big earthquake shooked the central region of Peru, causing 100 deaths. The maximum severity of shaking was reported in the Huacho city (VIII-IX MM). As coseismic effect a tsunami was generated, which flooded some villages and beach resorts, as Casma and Tortugas. From analysis and signal processing of three tidal records observed at stations of Chimbote, Callao and Marcona, we determined the parameters of the seismic source through an inversion process, in which the computed signal is compared with the observed signal by a non-negative least square process. The maximum slip is $4.7 \mathrm{~m}$ and is located in the Southern side of rupture geometry, which means that the maximum asperity or zone of greatest energy release is located under the Ocean in front of the Huacho city, this is consistent with the maximum intensity values reported. The scalar tsunami moment is $M_{0}=1.76 \times 10^{21} \mathrm{Nm}$, equivalent to a magnitude of $8.1 \mathrm{Mw}$.

Keywords: Earthquake, tsunami, inversion, numerical simulation.

Las formas de onda de un maremoto contienen información sobre los procesos de generación en la fuente sísmica y la propagación del maremoto que depende de la batimetría. Teniendo en cuenta la respuesta del instrumento, un antiguo mareógrafo mecánico y analógico de antes del año 1966, es posible realizar la inversiòn de las formas de onda del maremoto para obtener la distribución de las asperezas, zonas de mayor liberación de la energía sísmica, y la fuente sísmica.

\footnotetext{
*cjimenezt@unmsm.edu.pe
}

Una forma de verificar un modelo de fuente sísmica o modelo de deformación cosísmica inicial es mediante la simulación del maremoto correspondiente. Los parámetros tales como el tiempo de arribo, la amplitud y la polaridad de la primera onda y, en general, la forma de la onda del maremoto simulado deben tener una alta correlación con los correspondientes valores digitalmente procesados de los registros mareográficos para las estaciones disponibles [1].

Desde el punto de vista físico, el método de la inver- 
sión de ondas mareográficas, brinda un mejor resultado en comparación al método de inversión de ondas telesísmicas, en la obtención de los parámetros de la fuente sísmica, debido a que el modelo de velocidades es un parámetro que se conoce relativamente bien, puesto que la velocidad de las ondas del maremoto sólo depende de la batimetría. Por otro lado, en la inversión de las ondas telesísmicas no se conoce con certeza el modelo de velocidades de las ondas sísmicas, debido a las heterogeneidades locales de las propiedades físicas de la corteza. La bondad de los resultados obtenidos dependerá de la calidad de los registros mareográficos, de una buena cobertura azimutal de las estaciones y de la calidad y resolución de la batimetría en torno a las estaciones 2.

\section{Marco sismo-tectónico}

El terremoto de Huacho de 1966, se generó debido a la liberación de los esfuerzos tectónicos acumulados en la zona de interacción de las placas tectónicas de Nazca y Sudamérica. Estas dos placas convergen a una razón de 7-8 $\mathrm{cm} /$ año [3. El mecanismo focal de este sismo es del tipo de falla inversa en la interface entre las dos placas, con la placa de Nazca moviéndose por debajo de la placa continental, proceso conocido como subducción. La actividad sísmica dominante a lo largo de la costa del Perú, es una consecuencia directa del proceso de subducción de la placa de Nazca bajo la placa Sudamericana, por lo que la costa del Perú, tiene una historia de grandes terremotos.

Durante el siglo $X X$ y a inicios del siglo $X X I$ se han producido varios terremotos destructores de magnitud mayor o igual a $7.9 \mathrm{Mw}$ en la región central del Perú, entre la dorsal de Nazca y la fractura de Mendaña, como el de 1940 en Lima (8.0 Mw), de 1966 en Huacho (8.1 Mw), de 1970 en Ancash $(7.9 \mathrm{Mw})$; además sismos intraplaca con mecanismo focal de falla normal, ocurrió al interior de la placa de Nazca en 1974 en Cañete (8.1 Mw) y en el 2007 en Pisco $(8.1 \mathrm{Mw})$ [4]. Todos estos eventos sísmicos se han generado dentro del área de ruptura del gran sismo de Callao de $1746(9.0 \mathrm{Mw})$ [5].

El terremoto de Huacho de 1966, llenó parcialmente el gap sísmico dejado por el gran sismo de 1746; sin embargo, luego de 50 años de ocurrencia, es factible considerar a esta región de ruptura como un nuevo gap sísmico, debido a que los esfuerzos tectónicos no se relajaron completamente debido al sismo de 1966 y también debido a la acumulación de nuevos esfuerzos tectónicos en estos últimos 50 años. Chlieh et al. [6] sostiene que si se asume que la actual razón de cambio del déficit de momento sísmico es estacionaria, entonces el déficit de momento acumulado desde 1746 indica que la ocurrencia de un gran evento sísmico similar estaría atrasada. La acumulación del momento sísmico liberado por la secuencia de 1940-2007, representa sólo el $23 \%$ del déficit de momento acumulado desde 1746 , sugiriendo que una cantidad significativa de momento sísmico estaría aún por liberarse en este largo segmento de $500 \mathrm{~km}$ de la dorsal de Nazca a la fractura de Mendaña.

Como efecto cosísmico, el terremoto de Huacho, de 1966, generó un maremoto, que según el reporte de Silgado (7] narra que: a unos 50 minutos después del terremoto se produjo un tsunami moderado, registrándose la primera onda en los mareógrafos de la Punta, Chimbote y San Juan. En Casma y Tortugas hubo inundación, sufriendo grandes pérdidas varias fábricas pesqueras. La máxima intensidad (IX MM) ocurrió en la hacienda San Nicolás ubicada a 3 $\mathrm{km}$ al sur de Supe, en Huacho se reportó una intensidad de VIII MM y en Lima VII MM. También se reportó 100 víctimas mortales debido al sismo, pero ninguna debido al maremoto.

Por su parte, Lomnitz y Cabré [8] Ilevaron a cabo un trabajo de observación de campo, por encargo de la UNESCO, de los efectos del sismo y del maremoto. También explicaron los valores de los tiempos de arribo a las estaciones, indicando que la fosa peruana actúa como un canal de alta velocidad la cual refracta la energía de la onda hacia la costa.

\section{Adquisición y procesamiento digital de las señales mareográficas}

El registro mareográfico es una fuente importante de información que permite reproducir o caracterizar y validar los modelos numéricos de maremotos. La secuencia y forma sucesiva de las ondas, registradas en boyas en altamar, como las DART, y la forma del primer periodo de la onda para estaciones costeras proporcionan información sobre las características de la fuente sísmica, tales como la geometría de la zona de ruptura, la distribución de la dislocación o desplazamientos, entre otros.



Figura 1: Batimetría y topografía de la región central del Perú y distribución de las estaciones mareográficas utilizadas. 
Para llevar a cabo la presente investigación, se utilizaron las formas de onda del maremoto registradas en forma analógica, un trazo de lápiz sobre una hoja en un tambor giratorio, en una estación mecánica compuesta por un sistema de poleas y una boya flotante para medir el nivel del mar, el control del tiempo se realizaba con un reloj mecánico de cuerda, el cual era calibrado periodicamente, lo que implica una incertidumbre en el control del tiempo. Dichos registros mareográficos fueron obtenidos de la publicación de Murty y Wigen [9], los que fueron escaneados y digitalizados, y corresponden a tres estaciones mareográficas mecánicas pertenecientes a la Red Mareográfica Nacional ubicadas en los puertos de Chimbote, Callao y San Juan de Marcona, Figura 1, a cargo de la Dirección de Hidrografía y Navegación.

Para tener una buena distribución geométrica azimutal de las estaciones con respecto al epicentro, se debería disponer de más estaciones hacia el norte y al menos una boya en altamar, lo que no sucedió en este evento en particular, debido a la falta de más estaciones y registros de buena calidad y libre de ruido. Las estaciones de Talara, en el norte, y Matarani, en el sur, no registraron amplitudes importantes del maremoto.

Luego de un sismo tsunamigénico, la estación mareográfica registraría el evento y la señal correspondiente y estaría compuesta de un amplio espectro de frecuencias, con tres componentes principales: las mareas -de periodo largo-, el oleaje -de periodo corto-, y las ondas del maremoto. Para estudiar los registros mareográficos del maremoto se debe aplicar los algoritmos del procesamiento digital de señales [10].

En el caso de los registros analógicos, estos fueron escaneados y digitalizados. La elección de la frecuencia de muestreo de la señal depende de la calidad y resolución de la imagen escaneada y del algoritmo computacional utilizado para digitalizarlo. Una vez obtenida la serie de tiempo digitalizada, se interpolan los datos para una razón de muestreo de 1 minuto, por el método de interpolación cúbica, con el objetivo de homogenizar la razón de muestreo, debido a que cada estación puede tener una razón de muestreo diferente, de $1 \mathrm{~min}, 2 \mathrm{~min}$ o $5 \mathrm{~min}$. Luego, se remueve el valor medio de la señal para centrarlo con respecto al eje de las abscisas. Asimismo, se aplica un filtro Butterworth pasa-alta con $f_{c}=6.94 \times 10^{-5} \mathrm{~Hz}$ y un filtro pasa-baja con $f_{c}=0.0083 \mathrm{~Hz}$ para eliminar las componentes de periodo muy largo que corresponde a las mareas y las de periodo muy corto correspondiente a los oleajes. Finalmente, el tiempo se referencia con respecto al tiempo de origen del sismo [1].

Cabe recalcar que este fue el segundo maremoto que fue registrado por estaciones mareográficas locales, después del maremoto de Alaska de 1964. A grandes distancias, este evento fue registrado en estaciones ubicadas en la costa de California con amplitudes menores a $10 \mathrm{~cm}$.

\begin{tabular}{llccc}
\hline$N$ & Estación & $\begin{array}{c}\text { Lat } \\
\left(^{\circ}\right)\end{array}$ & $\begin{array}{c}\text { Lon } \\
\left(^{\circ}\right)\end{array}$ & $\begin{array}{c}\text { TA } \\
(\mathrm{min})\end{array}$ \\
\hline 1 & Chimbote & -09.0763 & -78.6127 & 66 \\
2 & Callao & -12.0710 & -77.1740 & 55 \\
3 & Marcona & -15.3467 & -75.1580 & 64 \\
\hline
\end{tabular}

Tabla 1: Las estaciones mareográficas están en orden correspondiente de norte a sur. El tiempo de arribo (TA) se mide con respecto al tiempo origen del sismo.

Para efectos computacionales del proceso de inversión, todos los registros se sincronizan de tal modo que el tiempo de arribo de la primera onda corresponde al minuto 55, un valor cercano al menor tiempo de arribo que corresponde a la estación de Callao, Tabla 1 . Se observa que el tiempo de arribo para la estación de Marcona es comparable con el de la estación de Chimbote, esto se debe al efecto de la batimetría de la fosa peruana y al fenómeno de refracción que controla la directividad de la propagación de las ondas del maremoto, tal como lo explicó Lomnitz y Cabré [8]. Con excepción de esta estación, los tiempos de arribo guardan cierta proporcionalidad con respecto a la distancia epicentral de cada estación.

Debido a que la velocidad y la directividad de las ondas del maremoto depende de la batimetría o topografía marina, es esencial contar con un buen modelo de batimetría para el cálculo de las funciones de Green o mareogramas elementales. La batimetría global se ha tomado del modelo GEBCO 30 [11], que posee una resolución de grilla de 30 segundos de arco que representa $\approx 927 \mathrm{~m}$, Figura 1 .

La batimetría en el norte del Perú cerca a Chimbote es poco profunda, casi superficial. La velocidad de las ondas del maremoto es proporcional a la raíz cuadrada de la batimetría, por lo que el tiempo de arribo de la primera onda a la estación de Chimbote no guarda una porporcionalidad con respecto a la ubicación del epicentro.

\section{Parámetros hipocentrales, mecanismo focal y ré- plicas}

Estos parámetros fueron calculados y reportados por varios autores, los que se resumen en la Tabla 2. Según Dewey y Spence [12], los parámetros hipocentrales fueron: Latitud $=-10.74^{\circ}$, Longitud $=-70.79^{\circ}$, y Profundidad $=21 \mathrm{~km}$ (Tabla 2). Kanamori [13] reportó una magnitud de $8.1 \mathrm{Mw}$. Abe [14] calculó la orientación del plano de ruptura o mecanismo focal a partir de la inversión de ondas telesísmicas, siendo estos parámetros: azimuth $=335^{\circ}$, buzamiento $=12^{\circ} \mathrm{y}$ ángulo de dislocación $=90^{\circ}$, Tabla 2 . Por lo tanto, el terremoto de 1966 representa un evento del tipo de falla inversa con un pequeño ángulo de buzamiento, cuyo ángulo de dislocacion es casi perpendicular al eje de la fosa marina.

Kelleher [15] recalculó la localización epicentral de las réplicas. Se ha contabilizado un total de 22 réplicas de profundidad superficial, menor a $60 \mathrm{~km}$, de magnitud mayor 
a $4.0 \mathrm{Mw}$, desde la ocurrencia del terremoto principal, 17 Oct 1966, hasta el 09 de diciembre del mismo año. También se reporta la ocurrencia de 2 réplicas de profundidad intermedia, las que no han sido graficadas en la Figura 2.

\begin{tabular}{ll}
\hline Fecha & 17 Oct 1966 \\
\hline Hora UTC & $21: 41: 51$ \\
Latitud & $-10.74^{\circ}$ \\
Longitud & $-78.79^{\circ}$ \\
Profundidad & $21 \mathrm{~km}$ \\
Magnitud & $8.1 \mathrm{Mw}$ \\
Azimuth & $335^{\circ}$ \\
Buzamiento & $12^{\circ}$ \\
Ángulo de dislocación & $90^{\circ}$ \\
\hline
\end{tabular}

Tabla 2: Los parámetros hipocentrales fueron reportados por Dewey and Spence [12]. La magnitud fue calculada por Kanamori [13] y el mecanismo focal fue calculado por Abe [14].

Las réplicas de profundidad focal superficial están distribuidas principalmente entre la fosa marina y la línea de la costa, a lo largo de la geometría de ruptura, aproximadamente desde la zona de Huacho hasta Casma abarcando una superficie con un área efectiva de alrededor de $200 \mathrm{~km}$ de largo por $120 \mathrm{~km}$ de ancho (Figura 2). Previamente, Dorbath et al. [16] le asignó una longitud de $120 \mathrm{~km}$ a la geometría de ruptura, luego utilizó este valor para obtener una ecuación de escalamiento de la magnitud con la dimensión del área de ruptura. Sin embargo, la distribución de las réplicas, Figura 2, implica un mayor tamaño del área de ruptura que en nuestras simulaciones hemos considerado.

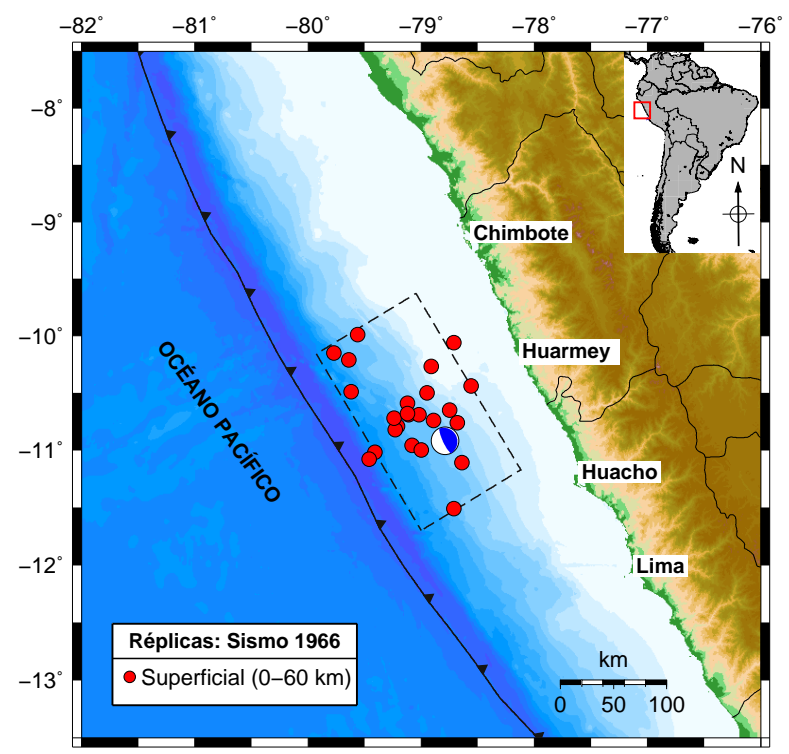

Figura 2: Réplicas del terremoto recalculadas por Kelleher [15]. Se observa la geometría del área de ruptura. El diagrama focal representa a la fuente sísmica.

\section{Método de Inversión}

Si se conocen los parámetros o las ecuaciones gobernantes que caracterizan a un sistema, las condiciones iniciales y de frontera, entonces se puede predecir el comportamiento de dicho sistema en cualquier instante de tiempo. A este tipo de problema, se le llama el problema directo. El problema inverso consiste en utilizar los resultados de ciertas observaciones para inferir los valores de los parámetros que caracterizan al sistema [17]. En este trabajo se utilizan los registros mareográficos del maremoto para deducir los parámetros de la fuente sísmica, la distribución de la dislocación o slip. El cálculo de la distribución de la fuente sísmica representa un típico problema inverso.

El proceso es considerado como un sistema lineal e invariante en el tiempo que posee dos propiedades importantes: a) Linealidad, que permite utilizar el principio de superposición para las funciones de Green y para las deformaciones unitarias. b) Invariancia en el tiempo, que permite realizar un desplazamiento en el tiempo de las señales observadas y sus correspondientes funciones de Green, sincronizando las señales observadas de forma que el tiempo de arribo del maremoto corresponda al minuto 55 , sólo para efectos de cálculo [1].

Este último punto hace posible la utilización de estaciones mareográficas independientemente de su distancia epicentral. La solución del problema inverso no es única, sino que se elige del conjunto de valores que proporcionen la mejor correlación entre los datos observados y los simulados.

Para estimar la distribución del slip, se utilizó el método de inversión para tres registros mareográficos de calidad aceptable [13]. El área de ruptura se dividió en doce subfuentes o fragmentos de idénticas dimensiones: $L=50 \mathrm{~km}$ y $W=40 \mathrm{~km}$, ubicados a una profundidad $H_{j}$ con respecto a la parte superior, según la coordenada vertical de cada sub-fuente. La relación entre los diferentes parámetros está dado por [1:

$$
\begin{aligned}
H_{j} & =h+\frac{W}{2} \operatorname{sen} \delta[1-\bmod (j, 2)], \quad j=1, \ldots, 8 \\
h & =h_{e}-\left[\left(x_{e}-x_{0}\right) \cos \Theta-\left(y_{e}-y_{0}\right) \sin \Theta\right] \tan \delta
\end{aligned}
$$

donde, $h$ es la profundidad de la parte superior del área de ruptura, $\left(x_{0}, y_{0}\right)$ son las coordenadas de la esquina inferior izquierda, $j$ representa la posición de la $j$-ésima sub-fuente, $\delta$ es el ángulo de buzamiento, $\bmod (j, 2)$ representa el módulo o resto de la división de $j$ con respecto a $2,\left(x_{e}, y_{e}, h_{e}\right)$ son las coordenadas del hipocentro o foco sísmico y $\Theta$ es el ángulo azimutal. Se debe notar que $H_{j}$ no es igual a la profundidad focal, pero están relacionados a través de la Ec.(2).

La deformación cosísmica se calculó para cada subfuente con un slip unitario, utilizando la formulación de Okada [18] y los datos del mecanismo focal. Los parámetros del mecanismo focal tales como azimuth, buzamiento 
y ángulo de dislocación son tomados de la investigación de Abe [14] y son iguales para cada una de las sub-fuentes (Tabla 2).

\section{Cálculo de las funciones de Green}

Desde el punto de vista físico, una función de Green representa la respuesta del sistema, señal simulada en la $i$ ésima estación, cuando dicho sistema es perturbado por un impulso unitario, una deformación debido a la dislocación unitaria en la $j$-ésima sub-fuente. Usando este campo de deformación como condición inicial, se calculan las formas de onda o funciones de Green en cada estación simulando la propagación del maremoto por el método de diferencias finitas para cada una de las sub-fuentes, usando una batimetría real. Las formas de onda mareográficas observadas son expresadas como una combinación lineal de las formas de ondas calculadas, en notación tensorial como,

$$
G_{i j}(t) m_{j}=d_{i}(t)
$$

donde $G_{i j}$ es la función de Green en la $i$-ésima estación generada por la $j$-ésima sub-fuente, $m_{j}$ es el valor del slip en la $j$-ésima sub-fuente y $d_{i}$ es la señal observada en la $i$-ésima estación.

Para obtener las funciones de Green correspondientes a las estaciones mareográficas de Chimbote, Callao y Marcona se utilizó una versión modificada del modelo numérico de propagación de maremotos TUNAMI para una sola grilla computacional [19]. En dicha grilla de propagación se utilizó un modelo lineal para una grilla batimétrica regular en coordenadas esféricas basado en la teoría lineal hidrodinámica de aguas someras. Las ecuaciones diferenciales de continuidad (4) y conservación de momentum lineal, son integradas numéricamente usando el método de diferencias finitas bajo el esquema numérico del "salto de rana". Se establece una condición de estabilidad numérica CFL menor a 0.8 para evitar inestabilidades numéricas.

$$
\begin{gathered}
\frac{\partial \eta}{\partial t}+\frac{\partial M}{\partial x}+\frac{\partial N}{\partial y}=0 \\
\frac{\partial M}{\partial t}+\frac{\partial}{\partial x}\left(\frac{M^{2}}{D}\right)+\frac{\partial}{\partial x}\left(\frac{M N}{D}\right)=-g D \frac{\partial \eta}{\partial x} \\
-\frac{g n^{2}}{D^{7 / 3}} M \sqrt{M^{2}+N^{2}} \\
\frac{\partial N}{\partial t}+\frac{\partial}{\partial x}\left(\frac{M N}{D}\right)+\frac{\partial}{\partial x}\left(\frac{N^{2}}{D}\right)=-g D \frac{\partial \eta}{\partial x} \\
-\frac{g n^{2}}{D^{7 / 3}} N \sqrt{M^{2}+N^{2}}
\end{gathered}
$$

donde

$$
\begin{aligned}
M & =\int_{-h}^{\eta} u d z \\
N & =\int_{-h}^{\eta} v d z \\
D & =\eta+h
\end{aligned}
$$

donde $M$ y $N$ representan la descarga de flujo para la dirección $x$ e $y$ respectivamente, $\eta$ es el nivel de agua y $h$ es la profundidad del agua con respecto al nivel medio del mar.

Las condiciones iniciales se basan en la teoría de deformación de Okada [18]. Las condiciones de frontera en la costa para el modelo lineal, grilla de propagación, suponen una pared vertical. El modelo numérico no toma en cuenta los efectos de dispersión, efectos no-lineales, que para este caso, por tratarse de un evento de campo cercano, no son apreciables.

La inversión se realizó mediante el método de mínimos cuadrados no-negativos [20]. Dicho método consiste en comparar la señal simulada con la señal observada y hacer mínimo el cuadrado del residual,

$$
\left|G_{i j} m_{j}-d_{i}\right|^{2} \rightarrow 0
$$

con la condición $m_{j}>0$. Adicionalmente, se ha impuesto una condición ad hoc para la subfuente 12 , la más cercana a Chimbote: $m_{12}=0$, un slip nulo, para un mejor control del tiempo de arribo a dicha estación. Sólo se debe tomar la parte de la señal correspondiente al primer periodo de la onda para evitar problemas de contaminación de la señal con efectos de reflexión y resonancias locales, debido a la batimetría y morfología de la costa alrededor de cada estación. En este caso, se tomó el intervalo de tiempo desde el minuto 55 al minuto 90 , para efectos de cálculo de la inversión.

\begin{tabular}{lcccc}
\hline$N$ & $\begin{array}{c}\text { Lat } \\
\left({ }^{\circ}\right)\end{array}$ & $\begin{array}{c}\text { Lon } \\
\left({ }^{\circ}\right)\end{array}$ & $\begin{array}{c}\text { Slip } \\
(\mathrm{m})\end{array}$ & $\begin{array}{c}H_{j} \\
(\mathrm{~km})\end{array}$ \\
\hline 1 & -11.7500 & -79.0800 & 1.60 & 03.50 \\
2 & -11.6013 & -78.7611 & 4.66 & 11.82 \\
3 & -11.4526 & -78.4422 & 3.64 & 20.13 \\
4 & -11.3425 & -79.2700 & 0.00 & 03.50 \\
5 & -11.1938 & -78.9511 & 3.43 & 11.82 \\
6 & -11.0451 & -78.6322 & 3.11 & 20.13 \\
7 & -10.9349 & -79.4601 & 2.11 & 03.50 \\
8 & -10.7862 & -79.1412 & 1.26 & 11.82 \\
9 & -10.6375 & -78.8223 & 0.64 & 20.13 \\
10 & -10.5274 & -79.6501 & 0.79 & 03.50 \\
11 & -10.3787 & -79.3312 & 0.76 & 11.82 \\
12 & -10.2300 & -79.0123 & 0.00 & 20.13 \\
\hline
\end{tabular}

Tabla 3: Distribución del slip para cada una de las 12 subfuentes. Las coordenadas corresponden a la esquina inferior izquierda y la profundidad corresponde al lado superior (según el eje vertical) de la fuente respectiva. 
El algoritmo garantiza que todos los valores de la dislocación o slip sean positivos, como una condición necesaria desde el punto de vista físico. Sin embargo, matemáticamente este modelo no proporcionará el resultado más óptimo con respecto al método de mínimos cuadrados simple. El resultado de la inversión se muestra en la Tabla 3.

Un método comunmente usado para definir la condición inicial del maremoto consiste en aplicar la misma deformación vertical del lecho marino a la superficie oceánica. Esto hace que el desplazamiento superficial inicial del mar sea una función de los parámetros de la fuente sísmica: dimensiones de la fuente, largo y ancho, profundidad, ángulo de buzamiento, azimut y ángulo de dislocación, epicentro y valores de la dislocación, slip. La pregunta es: ¿Sería la forma de onda del maremoto sensible a la variación de los parámetros de la fuente? La determinación de los parámetros que podrían afectar la forma de onda del maremoto mostraría la sensibilidad de las series de tiempo a los detalles de la fuente sísmica. Esto se realiza mediante una prueba de sensibilidad, mediante la cual se varìn ciertos parámetros y se compara la forma de onda del maremoto. Por ejemplo, el análisis de sensibilidad de Satake [21], muestra que el tamaño de la celda computacional debe ser menor que un octavo del tamaño de la fuente, para evitar problemas de dispersión numérica. También el espectro de frecuencias de la señal depende del tamaño de la fuente: si la fuente es grande, entonces el espectro contendrá componentes de baja frecuencia; si la fuente es pequeña, contendrá componentes de alta frecuencia.

\section{Resultados}

A partir de la distribución de los valores de la dislocación, slip, se obtiene el campo de deformación total como una combinación lineal de las deformaciones unitarias, el cual es utilizado como condición inicial de la propagación del maremoto. El máximo valor del slip es de $4.7 \mathrm{~m}$ y se ubica en la parte sur del área de ruptura, en la sub-fuente $\mathrm{N}^{\circ}$ 02. Esto explica el hecho de que la ciudad de Huacho haya sido la que sufrió el mayor impacto a causa del sismo, puesto que se encuentra frente a la sub-fuente 02. El máximo valor de la componente vertical de la deformación cosísmica inicial fue de $1.47 \mathrm{~m}$.

Debido a la posición del epicentro y a la geometría del área de ruptura, se puede inferir que el proceso de ruptura se propagó en forma bidireccional desde el epicentro hacia el norte y en menor grado hacia el sur. En torno al epicentro y en la región sur de la geometría de ruptura se concentra la mayor aspereza o zona de mayor liberación de energía sísmica, Figura 3. Esto concuerda con las máximas intensidades macrosísmicas de VIII a IX MM en Huacho, reportadas por Silgado [7].

De acuerdo a la investigación de Beck y Ruff 4], mediante una inversión de ondas telesísmicas, la aspereza prin- cipal está ubicada en torno al epicentro y hacia el sur-este a $80 \mathrm{~km}$ del epicentro se ubica otra aspereza más pequeña.

Para conciliar ambos resultados, la inversión de ondas telesísmica y la inversión de ondas de maremoto, se debería realizar una inversión combinada con los dos tipos de datos. Sin embargo, el patrón general sería el mismo: las mayores asperezas se ubicarían en torno al epicentro y hacia el sur del mismo.

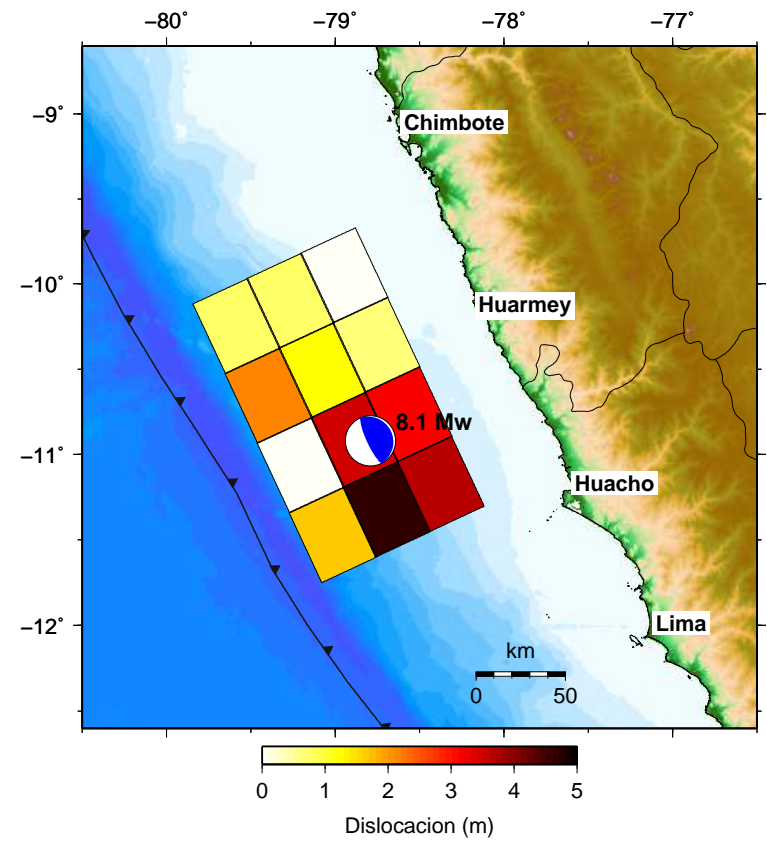

Figura 3: Se muestra la distribución de la fuente sísmica. Nótese la gran aspereza (color marrón oscuro) frente a la ciudad de Huacho.

\section{Momento sísmico escalar}

Teniendo la distribución de la dislocación o slip y las dimensiones de cada sub-fuente es posible calcular el momento sísmico escalar que se define como $M_{0}=\mu L W D$, donde $M_{0}$ es el momento sísmico, $\mu=4.0 \times 10^{10} \mathrm{~N} / \mathrm{m}^{2}$ es la rigidez promedio del medio elástico, $L=200 \mathrm{~km}$ longitud total de la fuente, $W=120 \mathrm{~km}$ es el ancho total de la fuente y $D=1.83 \mathrm{~m}$, es el slip promedio. El momento sísmico escalar total es $M_{0}=1.76 \times 10^{21} \mathrm{Nm}$, y según la relación $M_{w}=\frac{2}{3} \log \left(M_{0}\right)-6.07$, se obtiene una magnitud en la escala de momento de $8.1 \mathrm{Mw}$. Lo que significa que a partir de los registros mareográficos se obtiene un valor similar a la magnitud Mw calculada a partir de los registros sísmicos por Kanamori [13].

\section{Deformación y desplazamientos cosísmicos}

Como efecto cosísmico debido a la ocurrencia de un gran sismo en la zona de subducción, como el de Huacho 
en 1966, la corteza terrestre en el campo cercano se deforma de manera abrupta y permanente, formándose un patrón de zonas de levantamiento y hundimiento o subsidencia cosísmica. En general, si la fuente sísmica está en el mar lejos de la costa, entonces los puntos costeros sufrirán una subsidencia cosísmica; pero si la fuente sísmica está en la costa o muy cerca a la costa, entonces estos puntos podrían sufrir un levantamiento cosísmico, como ocurrió en Paracas, que se levantó alrededor de medio metro, durante el terremoto de Pisco 2007 [1].

En la Figura 4, se observa el patrón de deformación cosísmica de la corteza en la región de la fuente, el color rojo representa el levantamiento y el color azul representa la subsidencia cosísmica. Debido a la posición del área de ruptura, las ciudades de Huacho y Huarmey sufrieron una subsidencia cosísmica. Los vectores repesentan a la componente horizontal de desplazamiento cosísmico y tienen un comportamiento homogéneo apuntando en direccion Oeste, hacia el océano, llegando a una amplitud de más de 2 $\mathrm{m}$ cerca de la aspereza principal. Esto implica el desplazamiento cosísmico de las ciudades de Huacho y Huarmey en dirección al mar.

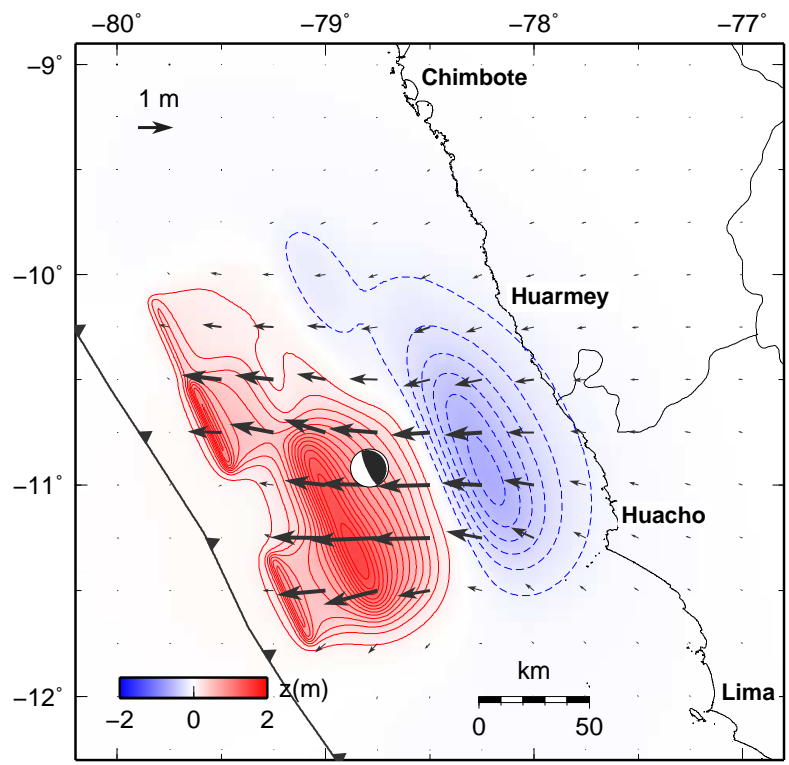

Figura 4: Se muestran los valores positivos de la componente vertical de la deformación cosísmica inicial en rojo y los valores negativos en azul. Los vectores representan la componente horizontal de desplazamiento cosísmico.

Este patrón de desplazamiento es consistente con la teoría del rebote elástico [22. En la fase intersísmica, los vectores de desplazamiento y velocidad apuntan en dirección Este hacia el continente; mientras que en la fase cosísmica, los esfuerzos tectónicos se relajan y el sistema rebota en la dirección contraria, hacia el mar, como se aprecia en la Figura 4.
Formas de onda de la inversión de la fuente sísmica

En la Figura 5, se puede observar los resultados de la inversión mediante la comparación de las ondas simuladas y observadas. Para efectos del cálculo de la inversión, todas las señales han sido desplazadas en el tiempo con respecto al tiempo de arribo a la respectiva estación, de forma que todos los tiempos de arribo están sincronizados en el minuto 55. Se observa que existe una buena correlación entre la onda observada y la onda calculada. Sin embargo, la falta de cobertura de más estaciones mareográficas en el Pacífico sur occidental, no permitió un mejor control en la obtención de los resultados. Se han obviado a las estaciones de Talara y Matarani debido a que la relación señal/ruido es muy pequeña.
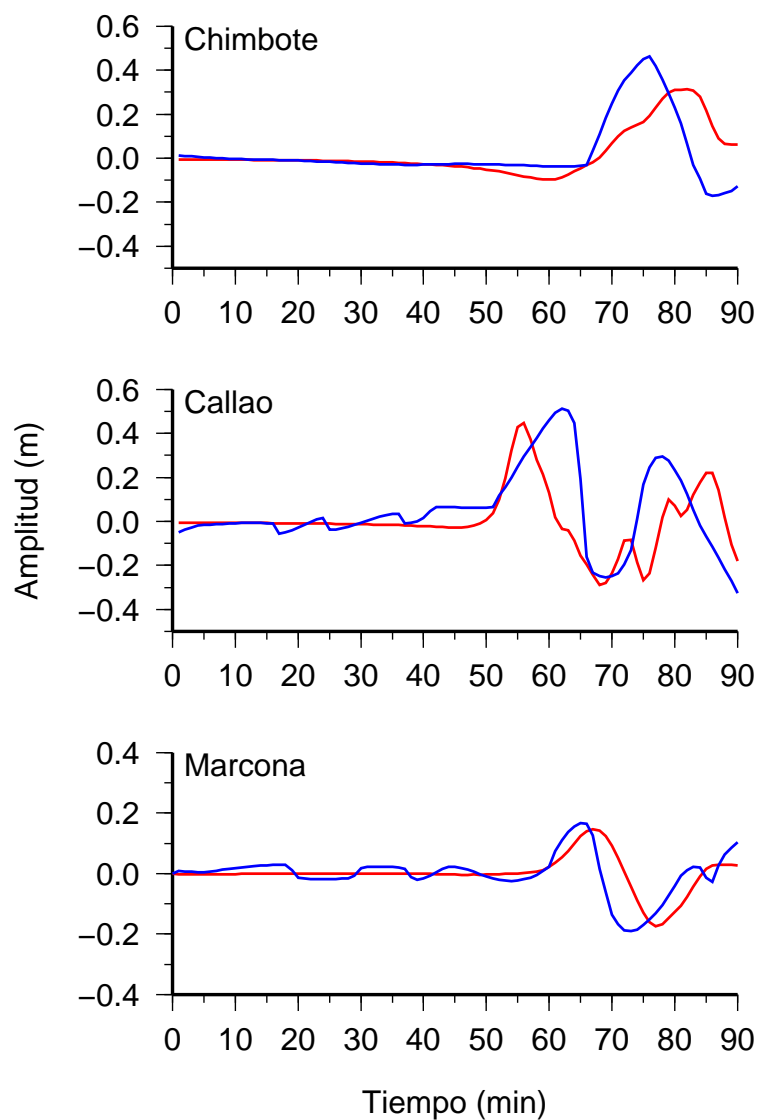

Figura 5: Se muestra los resultados de la inversión de los registros mareográficos para las diferentes estaciones. En color azul se muestra la señal observada y en color rojo la señal simulada. En las abcisas se muestra el tiempo en $\min y$ en las ordenadas la altura de las ondas en $\mathrm{m}$.

En la Tabla 4, se muestran los coeficientes de la correlación entre la señal observada y la señal simulada para cada estación. La estación de Marcona presenta el valor más alto de correlación, 0.59 . 


\begin{tabular}{llc}
\hline$N$ & Estación & Coeficiente de correlación \\
\hline 1 & Chimbote & 0.52 \\
2 & Callao & 0.39 \\
3 & Marcona & 0.59 \\
\hline
\end{tabular}

Tabla 4: El coeficiente de correlación entre las señales observadas y simuladas.

Para realizar la simulación de la fase de propagación del maremoto se utilizó el modelo numérico TUNAMI [19]. Este modelo integra las ecuaciones de la teoría lineal de aguas someras mediante el método de diferencias finitas centra- les, Figura 6. La condición inicial está dado por el campo de deformación cosísmico.

Con la fuente finalmente obtenida, se realizó nuevamente la simulación para el modelo no-lineal, después de realizar la inversión, esto para verificar que la señal obtenida con el proceso de inversión tiene correlación con la señal observada.

Con respecto a la directividad de las ondas del maremoto, la mayor cantidad de energía se dirigió en la dirección Oeste, es decir hacia la región de las islas Tonga-Kermadec. Esta directividad está determinada por la dirección perpendicular a la longitud del plano de la fuente y por la batimetría del Océano Pacífico.

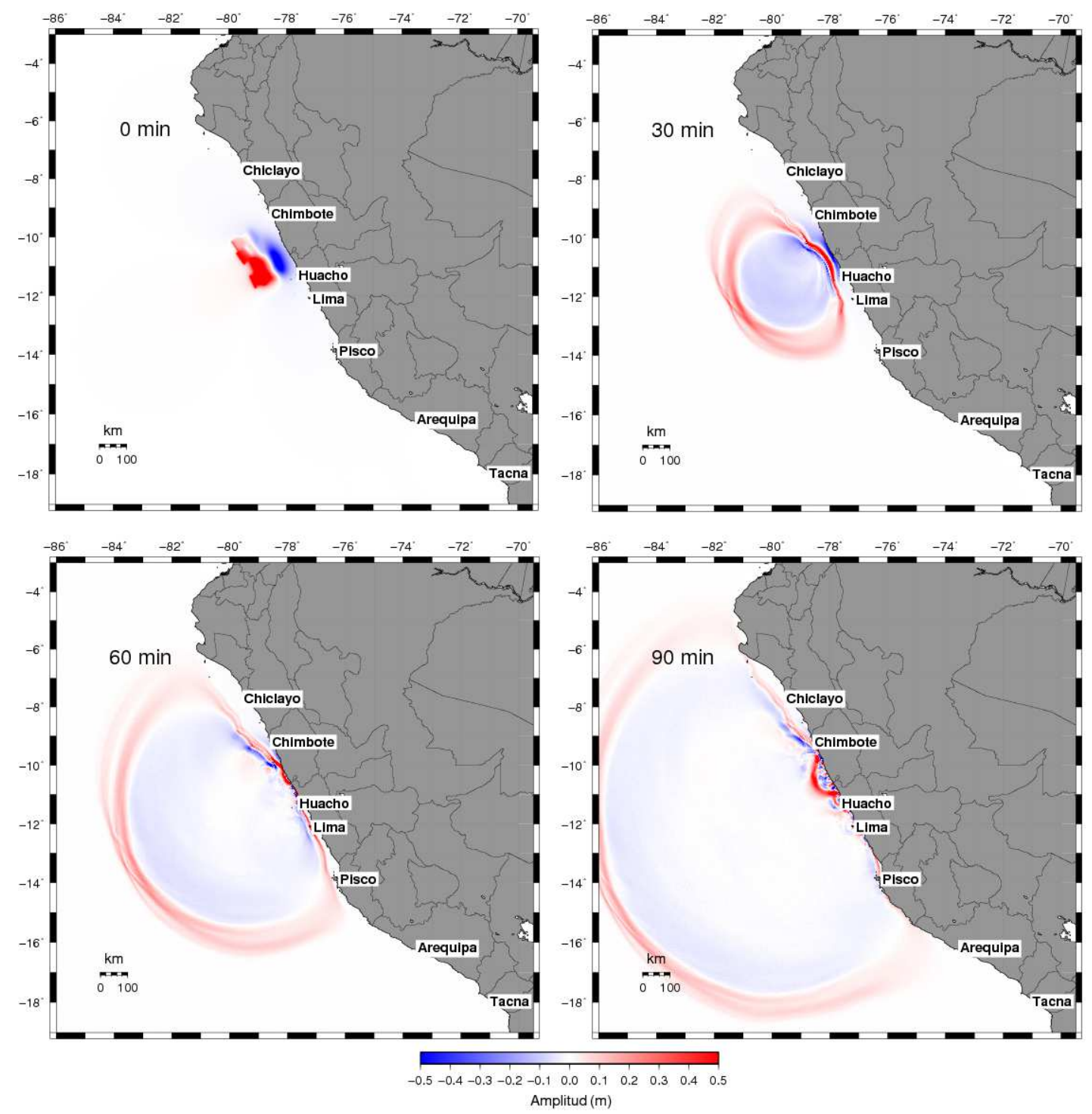

Figura 6: Propagación del maremoto para $t=0$ (condición inicial), 30, 60 y $90 \mathrm{~min}$. El color rojo representa los máximos valores de altura de ola $(m)$ mientras que el azul los valores mínimos. 


\section{Conclusiones}

La distribución de la fuente sísmica, obtenida en esta investigación, muestra una gran concentración de asperezas, zonas de mayor liberación de energía sísmica, en la parte sur de la geometría de ruptura frente a la ciudad de Huacho, lo que concuerda con los reportes de intensidad macrosísmica en dicha ciudad de VIII a IX MM.

La solución del problema inverso no es única, ya que se elige el conjunto de valores de dislocación que proporcionen la mejor correlación entre los datos observados y simulados, en base al método de los mínimos cuadrados no-negativos. La máxima amplitud de la deformación cosísmica inicial para este modelo es de $1.47 \mathrm{~m}$. La máxima dislocación es de $4.7 \mathrm{~m}$ y se ubica en la parte sur del área de ruptura específicamente, al frente de la ciudad de Huacho.

Para optimizar el resultado de la inversión debería utilizarse registros mareográficos adicionales, sin embargo las estaciones de Talara y Matarani no registraron amplitudes apreciables del evento. Adicionalmente, es importante tener una buena distribución azimutal de las estaciones mareográficas alrededor del área de ruptura. Por otro lado, el re- sultado dependerá de la calidad de los datos mareográficos, que en este caso no es muy buena, por ser registros analógicos de antiguos instrumentos mecánicos y de la calidad de la batimetría local alrededor de cada estación.

El proceso de ruptura, cuyo inicio fue en el foco o hipocentro, presentó una directividad bidireccional a lo largo del azimut en dirección al norte y en menor grado hacia el sur. El momento sísmico escalar calculado fue de $M_{0}=1.76 \times 10^{21} \mathrm{Nm}$ y la magnitud del terremoto inferido a partir de los registros mareográficos es de $8.1 \mathrm{Mw}$.

Una forma de verificar o validar el modelo de fuente sísmica propuesto es mediante la comparación de las señales mareográficas observadas y simuladas. En este sentido, el conjunto de las señales observada y simulada de la estación de San Juan de Marcona tuvo la mejor correlación, 0.59.

\section{Agradecimientos}

Se agradece a Cinna Lomnitz de la Universidad Autónoma de México por haber proporcionado material bibliográfico, por sus comentarios y sugerencias.

\section{Referencias}

[1] C. Jiménez, N. Moggiano, E. Mas, B. Adriano, Y. Fujii y S. Koshimura; J. Disast. Res. 9(6), 954 (2014).

[2] K. Satake y H. Kanamori; Natural Hazards 4, 193 (1991).

[3] E. Norabuena, L. Leffler, A. Mao, T. Dixon, S. Stein, S. Sacks, L. Ocola y M. Ellis; Science 279, 358 (1998)

[4] S. Beck y L. Ruff; Phys. Earth \& Planet. Int. 57, 199 (1989).

[5] C. Jiménez, N. Moggiano, E. Mas, B. Adriano, S. Koshimura, Y. Fujii y H. Yanagisawa J. Disast. Res. 8(2), 266-273 (2013).

[6] M. Chlieh, H. Perfettini, H. Tavera, J. Avouac, D. Remy, J. Nocquet, F. Rolandone, F. Bondoux, G. Gabalda y S. Bonvalot; J. Geophys. Res. 116(B12405), 21 (2011).

[7] E. Silgado; Historia de los sismos más notables ocurridos en el Perú (1513-1974), Instituto de Geología y Minería, Lima (1978).

[8] C. Lomnitz y R. Cabré; Bull. Seismol. Soc. Amer. 58, 645-661 (1968).

[9] T. Murty y S. Wigen; Tsunami Newsletter 8(1), 2-17 (1975).

[10] C. Jiménez, Rev. Inv. Fis. 10(2), 23 (2007).
[11] GEBCO; General Bathymetric Chart of the Oceans, http://www.gebco.net

[12] J. Dewey y W. Spence; Pageoph 117, 1148-1171 (1979).

[13] H. Kanamori; J. Geophys. Res. 82(20), 2981-2987 (1977).

[14] K. Abe; Phys. Earth \& Planet. Int. 5, 367-379 (1972).

[15] J. Kelleher; J. Geophys. Res. 27, 2087-2103 (1972).

[16] L. Dorbath, A. Cisternas y C. Dorbath; Bull. Seismol. Soc. Amer. 80(3), 515-576 (1990).

[17] A. Tarantola; Inverse problem theory and methods for model parameter estimation, Ed. Society for Industrial and Applied Mathematics, Philadelfia (2002).

[18] Y. Okada; Bull. Seismol. Soc. Am. 75(4), 1135 (1985).

[19] F. Imamura y S. Koshimura, Modelo numérico TUNAMI-N2, Tohoku University, Disaster Control Research Center, 2005.

[20] C. Lawson y R. Hanson; Solving least squares problems, Prentice-Hall, New York (1974).

[21] K. Satake; J. Phys. Earth. 35, 241-254 (1987).

[22] A. Udías, R. Madariaga y E. Buforn; Source Mechanism of Earthquakes: Theory and Practice, Cambridge University Press, 1st Edition, pp 302. United Kingdom (2014). 